\title{
Giant Faraday rotation in gadolinium doped silica optical fibers for high performance optical isolators
}

\author{
Rand Ismaeel $^{1 *}$, Ali Masoudi ${ }^{1}$, Yun Wang ${ }^{1}$, Wanvisa Talataisong ${ }^{1}$, Norberto Chiodini ${ }^{2}$, Timothy Lee ${ }^{1}$, \\ Martynas Beresna $^{1}$, Gilberto Brambilla ${ }^{1}$ \\ ${ }^{I}$ Optoelectronics Research Centre, University of Southampton, Southampton, SO17 1BJ, UK \\ ${ }^{2}$ Dipartimento di Scienza dei Materiali, Universita' di Milano -Bicocca, Via Roberto Cozzi 55, 20125 Milano, Italy \\ *rmni1g10@soton.ac.uk
}

\begin{abstract}
Silica fibres doped with small ( $<1 \mathrm{~mol} \%)$ concentrations of gadolinium ions have been fabricated and tested in a $1 \mathrm{~T}$ magnetic field showing a Faraday rotation two orders of magnitude larger than that reported in conventional telecom fibres. This is the largest Faraday rotation ever reported in low-loss optical fibers and could pave the way for a new class of optical isolators.

OCIS codes: 060.2340, 230.3240.For codes, see http://www.osapublishing.org/submit/ocis/
\end{abstract}

\section{Introduction}

Optical isolators are key components of transmission systems for avoiding any back-reflection induced instability or damage. They are used in conjunction with all types of lasers and mostly rely on the exploitation of the Faraday effect in crystals such as terbium gallium garnet (TGG) or bismuth iron garnet (BIG). The Faraday effect occurs in a material subjected to a magnetic field (Fig. 1), as a linearly polarized beam propagates along the direction of the magnetic field it experiences a rotation of the polarization azimuth [1].

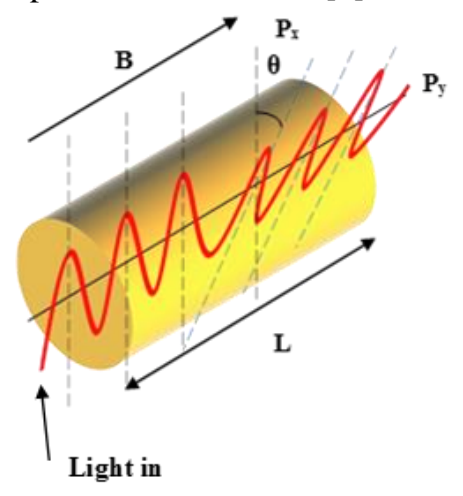

Fig.1 Faraday rotation: a linearly polarized beam in a magnetic field $B$ over an interaction length $l$ experiences a rotation of its polarization azimuth $\Delta \phi$.

The strength of the Faraday rotation is generally quantified with the Verdet constant V, defined as the degree of rotation $\Delta \phi$ per unit of magnetic field induction $\mathrm{B}$ along the beam propagation and unit of length $\mathrm{l}$ :

$$
\mathrm{V}=\frac{\Delta \phi}{B l}
$$

Most of the optical glasses investigated for Faraday effect were doped with rare-earths [2-4] such as terbium (Tb), which is widely considered as the best [5-6]. Concentrations of terbium as large as 50\% have been proposed to achieve Verdet constants one order of magnitude larger than that observed in standard telecom fibres. Here we show that concentrations of gadolinium as small as $0.1 \mathrm{~mol} \%$ can produce Faraday rotation that is two orders of magnitude larger than those reported in telecom optical fibres at $\lambda=1.55 \mu \mathrm{m}$. The optical isolators operating on gadolinium doped fibres could lead to new generation of fibre lasers.

\section{Fibre fabrication}

The optical fiber was fabricated using the rod-in-tube technique collapsing a commercial Heraeus fluorosilicate tube (cladding) on a silica rod doped with $0.1 \mathrm{~mol} \%$ of gadolinium fabricated via sol-gel [7] (core). Sol-gel reaction was created between tetramethoxysilane (TMOS) and gadolinium nitrates in a 4:1 solution of methanol:water. Gelation 
was carried out at $\mathrm{T}=45^{\circ} \mathrm{C}$ and the alcogel was dried into a xerogel and then densified under controlled atmosphere at $\mathrm{T}=1250{ }^{\circ} \mathrm{C}$. The cylindrical sample was thermally treated on the lathe and then pulled into a $500 \mu \mathrm{m}$ diameter cane, which was inserted into the fluorosilicate tube. The core-clad assembly was then pulled at $\mathrm{T} \sim 2000{ }^{\circ} \mathrm{C}$ in a graphite furnace, providing a fibre with a $125 \mu \mathrm{m}$ outer diameter and a numerical aperture of NA 0.05 . The fibre attenuation was estimated along a $200 \mathrm{~m}$ stretch of fibre to be in the region of $\sim 30 \mathrm{~dB} / \mathrm{km}$.

\section{Magneto-optic measurement}

A >1T magnetic field source was formed by injecting a 3A DC current into two hollow solenoids placed $\sim 10 \mathrm{~cm}$ apart (Fig. 2). A hollow iron core was inserted into the solenoids to provide a uniform magnetic flux. The optical fibre under test (FUT) was inserted into the iron core hole and maintained straight during the magnetic field measurements. The magnetic field intensity was measured in-situ with a Gauss meter.

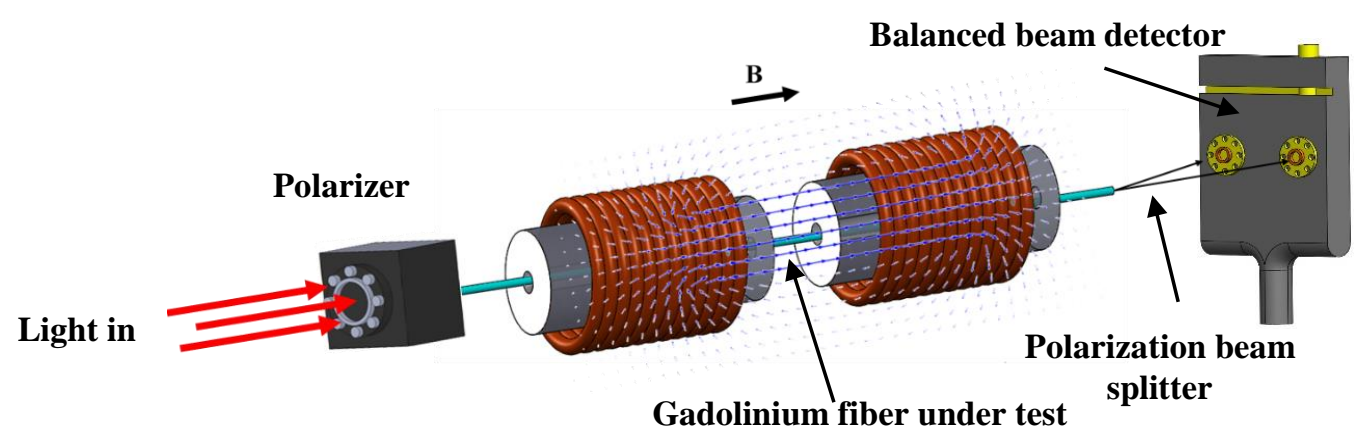

Fig. 2. Set-up used to generate $>1 \mathrm{~T}$ magnetic fields. The generated magnetic field is aligned with the coils axes. The fibre under test is inserted in the hollow metal core in the solenoids.

Changes in the azimuth of linearly polarized light $\Delta \phi$ were characterized using the set-up shown in fig. 3 and the Verdet constant was calculated using eq. 1 . Light at $\lambda=1.55 \mu \mathrm{m}$ was injected into a fiberized polarizer spliced to the FUT. The FUT was placed within the iron core of the measurement set-up and one of its pigtails spliced to a polarization beam splitter. The two orthogonally polarized beams were then directed to a balanced detector.

A telecom fibre (Corning SMF 28e) was used as reference for the polarization azimuth rotation measurements. On the telecom fibre subjected to a $1 \mathrm{~T}$ magnetic field, a rotation of $0.5^{\circ}$ was measured, corresponding to values of the Verdet constant consistent with those reported in the literature $(0.56 \pm 0.02 \mathrm{rad} / \mathrm{T} \cdot \mathrm{m}$ at $\lambda \sim 1.55 \mu \mathrm{m})$ [9]. The telecom fibre was then replaced with the Gd-doped fibre, which was spliced to the polarizer and to the polarization beam splitter. Three different $50 \mathrm{~mm}$ long sections were tested and all provided a rotation two orders of magnitude larger than that observed for telecom fibres, as shown in fig. 4.

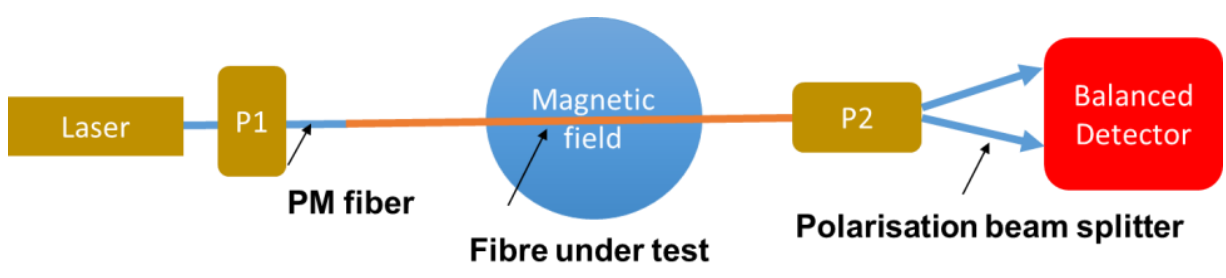

Fig. 3. Set-up used to measure Faraday rotation. Light from a laser source is injected into the fibre under test after passing a polarizer $\mathrm{P} 1$, and it is then split into the two orthogonal polarizations by a polarization beam splitter, connected to a balanced detector.

Further experiments were carried out with undoped fibres with similar guiding properties and showed a rotation consistent with that of a telecom fibre. The Gd-doped fibres were stripped of their coating to determine whether this might have any effect on the enhanced rotation but the magneto-optic response remained unaffected. Measurements at shorter wavelengths were carried out using a source delivering light at $\lambda \sim 1 \mu \mathrm{m}$. Initial results also showed over 
two times the improvement in rotation; however the stability of the system was limited as most of the optical components used were not optimized for operation at this wavelength.

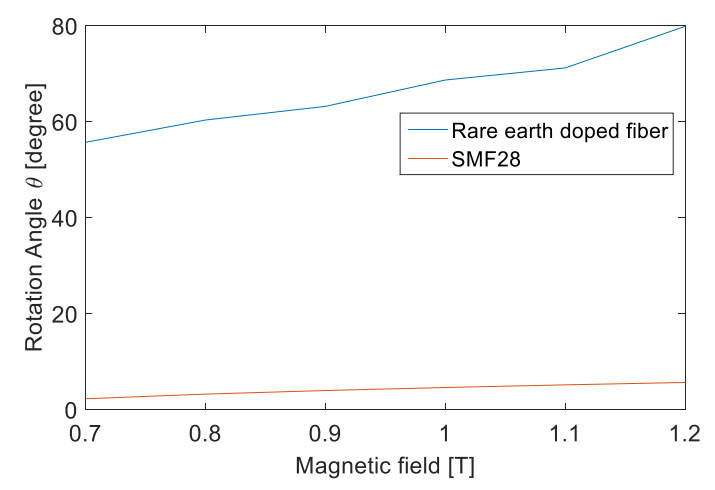

Fig. 4. The response of Gd fiber at different values of magnetic field.

In conclusion, a silica core fiber doped with small concentrations of gadolinium $(<1 \%)$ were fabricated and tested in $>1 \mathrm{~T}$ magnetic fields, showing polarization azimuth rotations $\sim 100$ times larger than that for standard telecom fibers at $\lambda \sim 1.55 \mu \mathrm{m}$ (Fig. 4). This is the largest value reported for low loss optical fibers and could pave the way for low cost isolators for applications in telecoms and high power fiber lasers.

\section{References}

[1] M. Yamane and Y. Asahara, Glasses for Photonics (Cambridge University Press, Cambridge, 2000).

[2] M. W. Shafer and J. C. Suits, "Preparation and Faraday Rotation of Divalent Europium Glasses," J. Am. Ceram. Soc. 49, 261-264 (1966).

[3] J. Ballato and F. E Snitzer, "Fabrication of fibers with high rare-earth concentrations for Faraday isolator applications," Appl. Opt. 34(30), 6848-6854 (1995).

[4] Y. Huang, H. Chen, W. Dong, F. Pang, J. Wen, Z. Chen, and T. Wang, "Fabrication of europium-doped silica optical fiber with high Verdet constant," Opt. Expr. 24 (16), 18709-18717 (2016).

[5] T. Hayakawa, T., Nogami, N. Nishi, and N. Sawanobori, "Faraday Rotation Effect of Highly

Tb2O3/Dy2O3-Concentrated B2O3-Ga2O3-SiO2-P2O5 Glasses," Chem. Mater. 14, 3223-3225 (2002).

[6] J. Qiu, K. Tanaka, N. Sugimoto and N. Hirao, "K. Faraday effect in Tb3+-containing borate, fluoride and fluorophosphate glasses,” J. Non-Cryst. Solids 213-214, 193-198 (1997).

[7] A. Vedda, N. Chiodini, D. Di Martino, M. Fasoli, M. Martini, F. Moretti, E. Rosetta, G. Spinolo, M. Nikl, N. Solovieva, A. Baraldi and R. Capelletti, "Luminescence properties of rare-earth ions in SiO2 glasses prepared by the sol-gel method," J. Non-Cryst. Sol. 345-346, 338-342 (2004).

[8] J. Townsend, S. Poole, and D. Payne, "Solution-doping technique for fabrication of rare-earth-doped optical fibres," Electron. Lett. 23, 329 (1987).

[9] Y. Huang, L. Chen, Q. Guo, F. F. Pang, J. X. Wen, J. X., Y. N. Shang, and T.Y. Wang, "The Measurement System of Birefringence and Verdet Constant of Optical Fiber," 2013 International Conference on Optical Instruments and Technology: Optoelectronic Measurement Tech. and Systems, Proc. SPIE, 9046, 904615-1(2013). 